\title{
GNSS Signal Processing with EKF and UKF for Stationary User Position Estimation
}

\author{
MERT SEVER \\ Hezârfen Aeronautics and Astronautics Technologies Institute for Space Sciences \\ Turkish National Defense University \\ Yeşilyurt, 34149, Bakırköy, Istanbul \\ TURKEY \\ CHINGIZ HAJIYEV \\ Faculty of Aeronautics and Astronautics \\ Istanbul Technical University \\ Ayazağa, 34469, Maslak, Istanbul \\ TURKEY
}

\begin{abstract}
Precise and accurate estimation of state vectors is an important process during position determination. In this study, Extended Kalman Filter (EKF) and Unscented Kalman Filter (UKF) of stationary user, state vectors defined in Earth Centered Inertial (ECI) coordinate system, accompanied by GNSS measurement data. It is aimed to make estimations with methods. EKF and UKF methods were compared with each other. In this study, the effects of nonlinear motion analysis and linearization methods on state vector estimations were investigated. Thanks to this study, estimations of the positioning information required during the specific tasks of many moving platforms have been made.
\end{abstract}

Keywords: EKF, UKF, GNSS, Positioning, Kalman Filter

Received: January 15, 2021. Revised: July 26, 2021. Accepted: August 23, 2021. Published: August 31, 2021.

\section{Introduction}

Precise positioning is important to obtain the correct location, and the use of a Global Satellite Navigation System (GNSS) receiver is becoming widespread in the military and civilian areas [10]. Although GNSS receivers are developed, there may be situations where sufficient sensitive measurement cannot be obtained. For these cases, GNSS measurement accuracy can be improved by using mathematical filters such as the Kalman method.

In the study of Soken and Hajiyev attitude estimation was made for pico satellites using the Unscented Kalman Filter [14]. In the aforementioned study, the measurement parameters obtained by modeling magnetometer and speed gyroscope were approximated to the real values with the help of UKF.

Another study by Kumar et al, GNSS-based position estimation was performed using EKF [9]. In this study, in which EKF is applied for the stationary user, it has been shown that the EKF approach gives high accuracy results in estimating position and clock bias.

In this study, 4 GNSS satellites were chosen to estimate the position of Şükrü SARAÇOĞLU Stadium (Istanbul/Turkey). Earth Centered Inertial
(ECI) reference frame were used. Position estimation was obtained using the pseudo-ranging method. Clock bias and random errors were added to the distances between the GNSS satellites and the user for simulating GNSS receiver errors. Here random is white gaussian random noise with zero mean and unit variance.

It's obtained from study that estimated position with Kalman Filters are more accurate than measurement. Filter results converges to actual state conditions in short-time interval.

\section{Problem Statement}

In this study, by using 4 GNSS satellites, Şükrü Saraçoğlu Stadium coordinates were estimated with the use of pseudo-ranging approach. Obtained noisy measurement distances by GNSS receiver, processed with UKF and EKF for increasing the accuracy of the estimation

$$
\begin{aligned}
& D_{a(i)}=\sqrt{\left(x_{i}-x\right)^{2}+\left(y_{i}-y\right)^{2}+\left(z_{i}-z\right)^{2}} \\
D_{(i)}= & \sqrt{\left(x_{i}-x\right)^{2}+\left(y_{i}-y\right)^{2}+\left(z_{i}-z\right)^{2}}+b_{i}+v_{i} \\
L_{i}= & \sqrt{x_{i}^{2}+y_{i}^{2}+z_{i}^{2}}
\end{aligned}
$$




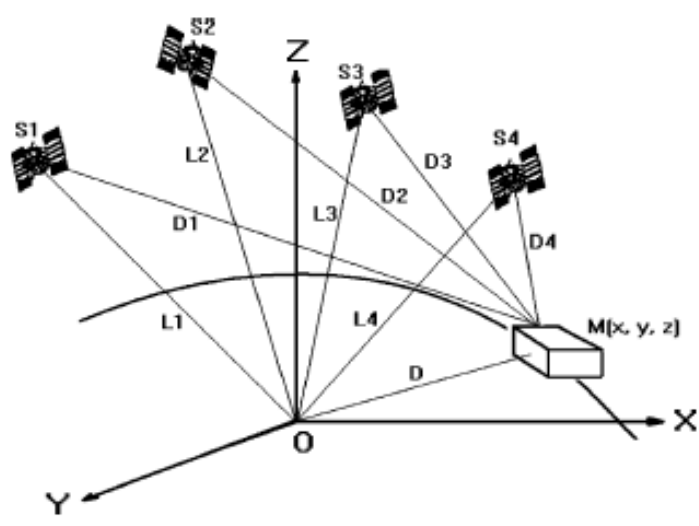

Fig. 1. Distance measurement scheme [6]

Here $x_{i}, y_{i}, z_{i}$ represents the Descartes coordinates of the $i$ th GPS satellite, $x, y, z$ represents the coordinates of the user location, $v_{i}$ is white gaussian noise with zero mean and $b$ is the clock bias. $L_{1}, L_{2}, L_{3}$ and $L_{4}$ are distance between origin and GPS satellite. $D_{a}$ is the actual distance and $D$ is the measured distance by GPS receiver. After calculating actual distance with Eq.(1), random zero mean gaussian errors and clock bias were added to actual distances for making model more realistic. This calculation simulates the signal from the GPS receiver.

The continuous-time state equation for simulated stationary user model is given as:

$$
\begin{aligned}
\dot{x} & =0 \\
\dot{y} & =0 \\
\dot{z} & =0 \\
\dot{b} & =u_{s}
\end{aligned}
$$

where $u_{s}$ is the white noise with zero mean.

The state equation can be written in the discretetime form as:

$$
\begin{gathered}
x(k)=x(k-1) \\
y(k)=y(k-1) \\
z(k)=z(k-1) \\
b(k)=b(k-1)+T_{s} u_{s}
\end{gathered}
$$

where $T_{s}$ is the sampling time.

If we rewrite the preceding statements for scalar equations,

$$
\left[\begin{array}{l}
x(k) \\
y(k) \\
z(k) \\
b(k)
\end{array}\right]=\left[\begin{array}{llll}
1 & 0 & 0 & 0 \\
0 & 1 & 0 & 0 \\
0 & 0 & 1 & 0 \\
0 & 0 & 0 & 1
\end{array}\right]\left[\begin{array}{c}
x(k-1) \\
y(k-1) \\
z(k-1) \\
b(k-1)
\end{array}\right]+\left[\begin{array}{c}
0 \\
0 \\
0 \\
T_{s} u_{s}
\end{array}\right]
$$

This means that the systems dynamics matrix is unit:

$$
\phi(k, k-1)=\left[\begin{array}{cccc}
1 & 0 & 0 & 0 \\
0 & 1 & 0 & 0 \\
0 & 0 & 1 & 0 \\
0 & 0 & 0 & 1
\end{array}\right]
$$

The system noise vector is:

$$
W(k)=\left[\begin{array}{c}
0 \\
0 \\
0 \\
T_{s} u_{s}
\end{array}\right]
$$

The state space model can be written in matrix form as

$$
\begin{aligned}
X(k) & =\phi(k, k-1) X(k-1)+W(k) \\
X(k) & =\left[\begin{array}{llll}
x(k) & y(k) & z(k) & b(k)
\end{array}\right]^{T}
\end{aligned}
$$

Here $X(k)$ is the state vector.

\subsection{Extended Kalman Filter}

Extended Kalman Filter (EKF) is an approach for non-linear systems. This approach linearizes the system and estimates the state vectors. Unlike the Linear Filter, the covariances and gain matrix must be computed continuously as estimations and predictions are made. The Extended Kalman Filter uses a linearized model that needs to be calculated from approximate state information. Therefore, the filter must be well positioned initially for linearized models to be valid. State vector of the model were given in Eq. (8).

Estimation Equation:

$$
\hat{X}(k)=\hat{X}(k-1)+K(k) \hat{Z}(k / k-1)
$$

Extrapolation equation:

$$
\hat{X}(k / k-1)=\hat{X}(k-1 / k-1)
$$

Innovation sequence:

$$
\hat{Z}(k / k-1)=D(k)-\bar{D}(k)
$$

$$
D(k)=\left[\begin{array}{c}
D_{1}(k) \\
D_{2}(k) \\
D_{3}(k) \\
D_{4}(k)
\end{array}\right] ; \quad \bar{D}(k)=\left[\begin{array}{c}
\bar{D}_{1}(k) \\
\bar{D}_{2}(k) \\
\bar{D}_{3}(k) \\
\bar{D}_{4}(k)
\end{array}\right]
$$

$$
\bar{D}_{i}=\sqrt{\begin{array}{c}
\left(x_{i}(k)-\hat{x}(k-1)\right)^{2}+\left(y_{i}(k)-\hat{y}(k-1)\right)^{2} \\
+\left(z_{i}(k)-\hat{z}(k-1)\right)^{2} \\
+\hat{b}(k-1)
\end{array}}
$$

Kalman gain coefficient matrix:

$$
K(k)=P(k / k-1) H(k)^{T}\left[H(k) P(k / k-1) H(k)^{T}+R(k)\right]^{-1}
$$

Predicted correlation matrix of estimation error

$$
P(k / k-1)=\phi(k, k-1) P(k / k-1) \phi(k, k-1)^{T}+Q(k-1)
$$

Estimated correlation matrix of estimation error:

$$
P(k / k)=P(k-1 / k-1)-K(k) H(k) P(k-1 / k-1)
$$

The system noise covariance matrix is taken as:

$$
Q(k-1)=0,01 \times I_{4 \times 4}
$$

Measurement Matrix: 


$$
H(k)=\left[\begin{array}{llll}
\frac{\partial D_{1}}{\partial_{x}} & \frac{\partial D_{1}}{\partial_{y}} & \frac{\partial D_{1}}{\partial_{z}} & \frac{\partial D_{1}}{\partial_{b}} \\
\frac{\partial D_{2}}{\partial_{x}} & \frac{\partial D_{2}}{\partial_{y}} & \frac{\partial D_{2}}{\partial_{z}} & \frac{\partial D_{2}}{\partial_{b}} \\
\frac{\partial D_{3}}{\partial_{x}} & \frac{\partial D_{3}}{\partial_{y}} & \frac{\partial D_{3}}{\partial_{z}} & \frac{\partial D_{3}}{\partial_{b}} \\
\frac{\partial D_{4}}{\partial_{x}} & \frac{\partial D_{4}}{\partial_{y}} & \frac{\partial D_{4}}{\partial_{z}} & \frac{\partial D_{4}}{\partial_{b}}
\end{array}\right]
$$

Measurement error covariance matrix $(\mathrm{R})$ :

$$
R(k)=\left[\begin{array}{cccc}
\sigma^{2} & 0 & 0 & 0 \\
0 & \sigma^{2} & 0 & 0 \\
0 & 0 & \sigma^{2} & 0 \\
0 & 0 & 0 & \sigma^{2}
\end{array}\right]
$$

Here $\sigma$ is the standard deviation of distance measurement error and $\sigma_{D 1}=\sigma_{D 2}=\sigma_{D 3}=\sigma_{D 4}=$ $\sigma=10 \mathrm{~m}$.

\subsection{Unscented Kalman Filter}

The Unscented Kalman Filter is an approach that creates a state vector into a set of state vector. Despite the EKF, for a model with $\mathrm{n}$ state vectors, UKF generates $2 n+1$ conditions, then, at that point estimates the new state for each condition and takes the average [7].

Let's assume that $X_{0}$ is the initial condition of the user,

$$
\begin{gathered}
X_{0}(k / k)=\hat{X}(k / k) \\
X_{i}(k / k)=\hat{X}(k / k)+(\sqrt{(n+k)[P(k / k)+Q(k)]})_{i} \\
X_{i+n}(k / k)=\hat{X}(k / k)-(\sqrt{(n+k)[P(k / k)+Q(k)]})_{i}
\end{gathered}
$$

Here, $X_{0}(k / k), X_{i}(k / k)$ and $X_{i+n}(k / k)$ are created sigma state conditions, $\mathrm{Q}(\mathrm{k})$ system noise vector and $\kappa$ scaling parameter and $\mathrm{n}$ is the number of states. In this study, because of the model is stationary $n=4$. These 4 conditions are 3 axis position $(\mathrm{x}, \mathrm{y}, \mathrm{z})$ and clock bias (b). By considering previous studies, sum of the $n$ and $\kappa$ chosen as $3(n+\kappa=3)$ [14, $10]$.

Estimation equation:

$$
X(k+1 / k)=f(X(k / k), k)
$$

Assuming the model is stationary,

$$
X(k+1 / k)_{i}=X(k / k)_{i}
$$

Obtained estimation used for calculation of predicted mean and covariance.

$$
\hat{X}(\mathrm{k}+1 / \mathrm{k})=\frac{1}{n+\kappa}\left[\kappa X_{0}(k+1 / k)+\frac{1}{2} \sum_{i=1}^{2 n} X_{i}(k+1 / k)\right]
$$

$$
\begin{aligned}
P(k+1 / k)=\frac{1}{n+\kappa} & \left\{\kappa\left[X_{0}(k+1 / k)-\hat{X}(k+1 / k)\right]\right. \\
& \times\left[X_{0}(k+1 / k)-\hat{X}(k+1 / k)\right]^{T} \\
& +\frac{1}{2} \sum_{i=1}^{2 n}\left[X_{i}(k+1 / k)-\hat{X}(k+1 / k)\right] \\
& \left.\times\left[X_{i}(k+1 / k)-\hat{X}(k+1 / k)\right]^{T}\right\}
\end{aligned}
$$

Here $\hat{X}(k+1 / k)$ is the predicted mean and $P(k+1 / k)$ is the predicted covariance matrices. Predicted observation and predicted observation covariance matrix can be shown as,

$$
\begin{aligned}
& \hat{Y}(k+1 / k)=\frac{1}{n+\kappa}\left\{k Y_{0}(k+1 / k)+\frac{1}{2} \sum_{i=1}^{2 n} Y_{i}(k+1 / k)\right\} \\
& \text { where } Y_{i}(\mathrm{k}+1 / \mathrm{k})=h\left[\hat{X}_{i}(\mathrm{k}+1 / \mathrm{k})\right], \\
& \qquad \begin{aligned}
& P_{y y}(k+1 / k)=\frac{1}{n+\kappa}\left\{\kappa\left[Y_{0}(k+1 / k)-\hat{Y}(k+1 / k)\right]\right. \\
& \times\left[Y_{0}(k+1 / k)-\hat{Y}(k+1 / k)\right]^{T} \\
&+\frac{1}{2} \sum_{i=1}^{2 n}\left[Y_{i}(k+1 / k)\right. \\
&-\hat{Y}(k+1 / k)] \\
&\left.\times\left[Y_{i}(k+1 / k)-\hat{Y}(k+1 / k)\right]^{T}\right\} \\
&
\end{aligned}
\end{aligned}
$$

Innovation covariance can be expressed as following equation

$$
P_{v v}(k+1 / k)=P_{y y}(k+1 / k)+R(k+1)
$$

In this equation $R(k+1)$ is the covariance matrix of measurement error. By using the following equation cross covariance matrix can be obtained

$$
\begin{aligned}
P_{x y}(k+1 / k)=\frac{1}{n+} & \left\{\kappa\left[X_{0}(k+1 / k)-\hat{X}(k+1 / k)\right]\right. \\
& \times\left[Y_{0}(k+1 / k)-\hat{Y}(k+1 / k)\right]^{T} \\
& +\frac{1}{2} \sum_{i=1}^{2 n}\left[X_{i}(k+1 / k)\right. \\
& -\hat{X}(k+1 / k)] \\
& \left.\times\left[Y_{i}(k+1 / k)-\hat{Y}(k+1 / k)\right]^{T}\right\}
\end{aligned}
$$

The innovation sequence of UKF can be written as

$$
e(k+1)=\mathrm{D}(k+1)-\hat{Y}(k+1 / k)
$$

where $D(k+1)$ are the distance measurements.

Kalman gain matrix is,

$$
K(k+1)=P_{x y}(k+1 / k) P_{v v}{ }^{-1}(k+1 / k)
$$

As a final step, updated states and covariance matrix obtained as,

$$
\begin{gathered}
\hat{X}(k+1 / k+1)=\hat{X}(k+1 / k)+K(k+1) e(k+1) \\
P(k+1 / k+1)=P(k+1 / k)-K(k+1) P_{v v}(k+1) K^{T}(k+1)
\end{gathered}
$$

When the equations shown above are applied to the model continuously, the filter estimation will occur in the loop [14]. 


\section{Simulation Results and Discussion}

In this study, a stationary user location estimated. Obtained GNSS data were processed with Unscented and Extended Kalman Filters. At the end of the simulations these two methods were compared.

By analyzing the graphs, its obtained that both EKF and UKF estimations are less faulty than direct measurement. Approaches, decreases the measurement errors and estimations converges to the actual state conditions by time.

Figure 2, 3, 4 and 5 shows the $\mathrm{x}, \mathrm{y}, \mathrm{z}$ position and clock bias estimations respectively. The blue line represents the EKF estimation and red line represents the actual position of the user. Also, errors of the estimations and variances of the estimation errors were given below for each position axis and clock bias.
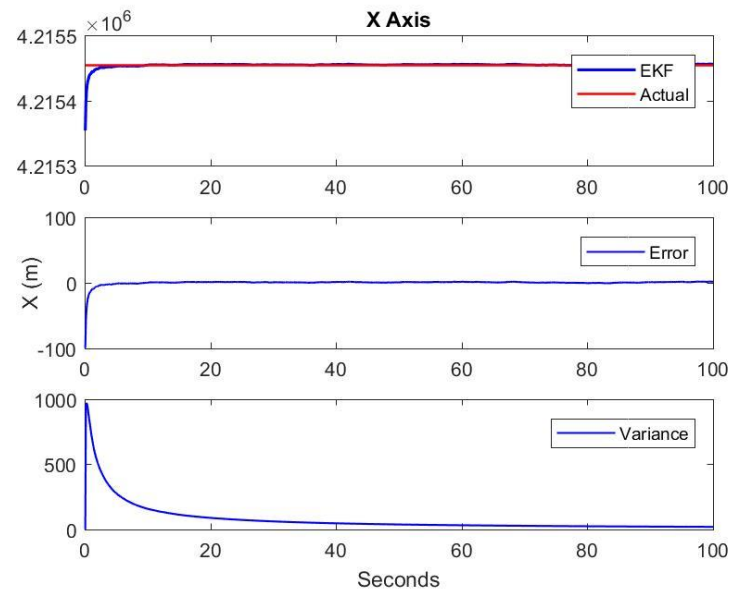

Fig.2: X Axis Position Estimation via EKF
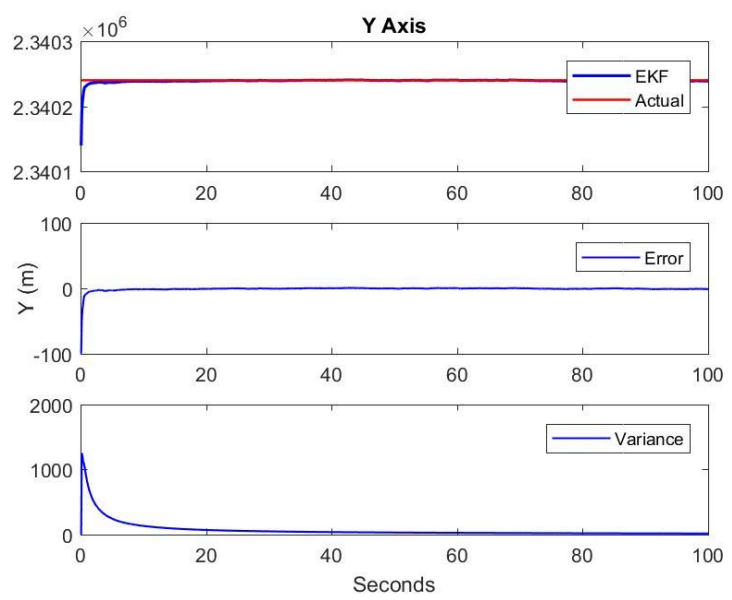

Fig.3: Y Axis Position Estimation via EKF
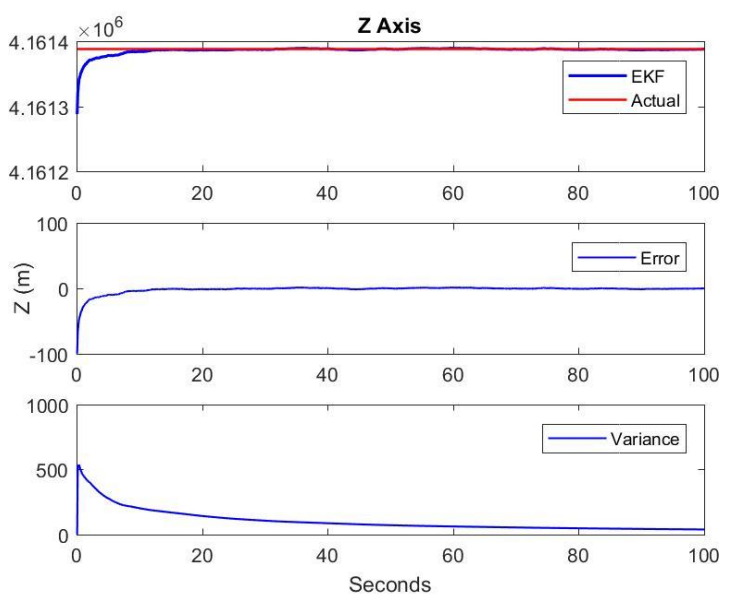

Fig.4: Z Axis Position Estimation via EKF
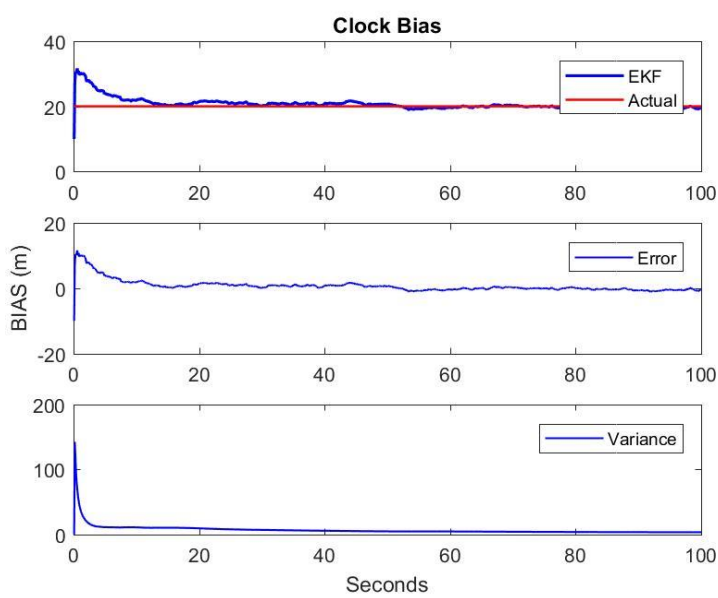

Fig.5: Clock Bias Estimation via EKF

Figure 5 shows the normalized innovation graphs. Normalized innovation represents the characteristic of the filter. It's expected that graph should be noisy around zero. From figure 5 its obtained that filter works properly. In the graphs, 1,2,3,4 indicates innovation that is consistent with GNSS measurements.

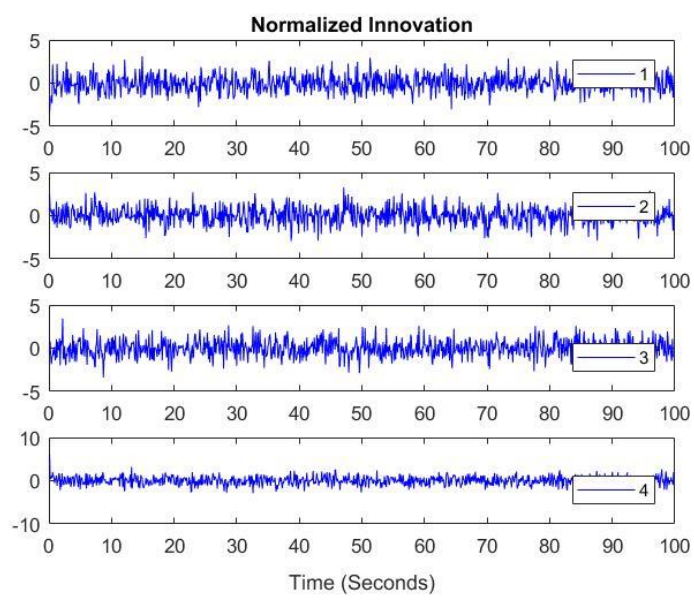

Fig.6: EKF Normalized Innovation Graphs 
Figure 7, 8, 9 and 10 shows the $\mathrm{x}, \mathrm{y}, \mathrm{z}$ position and clock bias estimations of the UKF respectively. The blue line represents the UKF estimation and red line represents the actual position of the user. Also, errors of the estimations and variances of the estimation errors were given below for each position axis and clock bias.
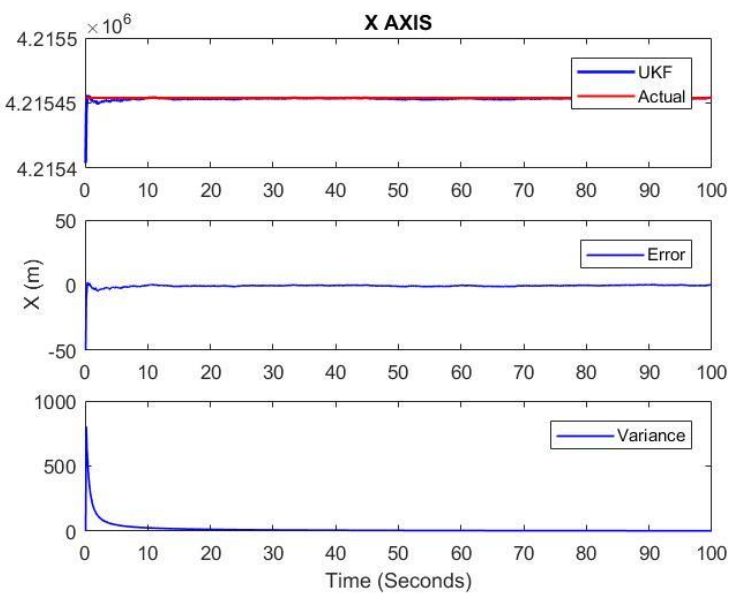

Fig.7: X Axis Position Estimation via UKF
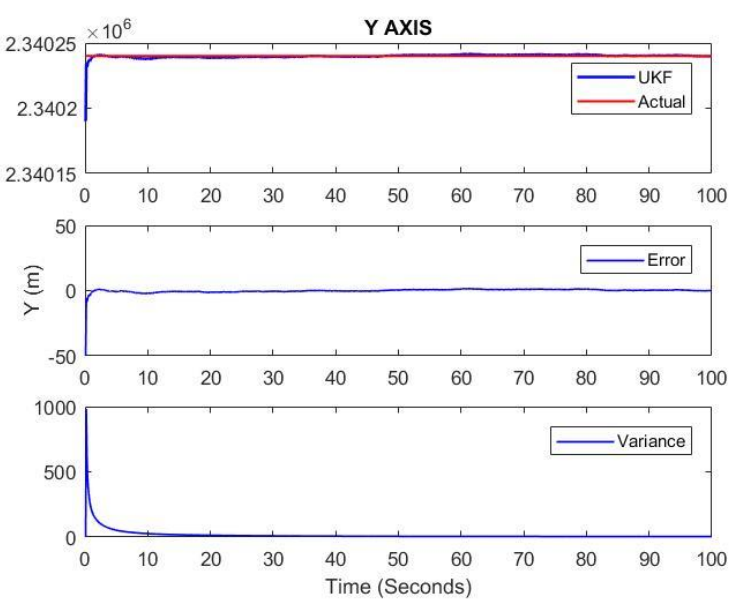

Fig.8: Y Axis Position Estimation via UKF
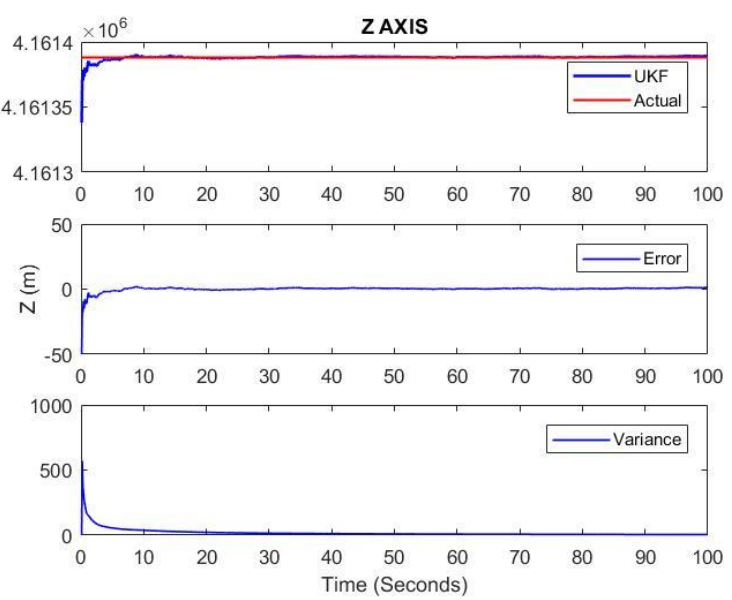

Fig.9: Z Axis Position Estimation via UKF
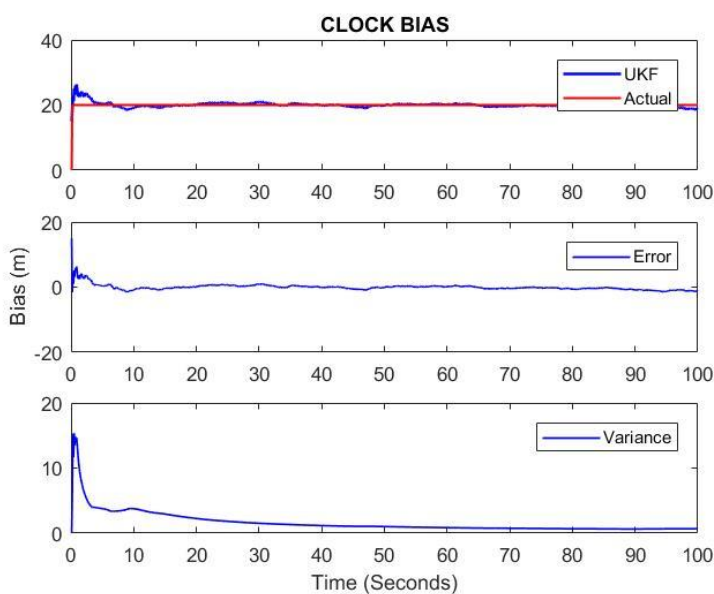

Fig.10: Clock Bias Estimation via UKF

Figure 11 shows the normalized innovation graphs of the UKF. From the figure it's obtained that filter is working properly. In the graphs, 1,2,3,4 indicates innovation that is consistent with GNSS measurements.

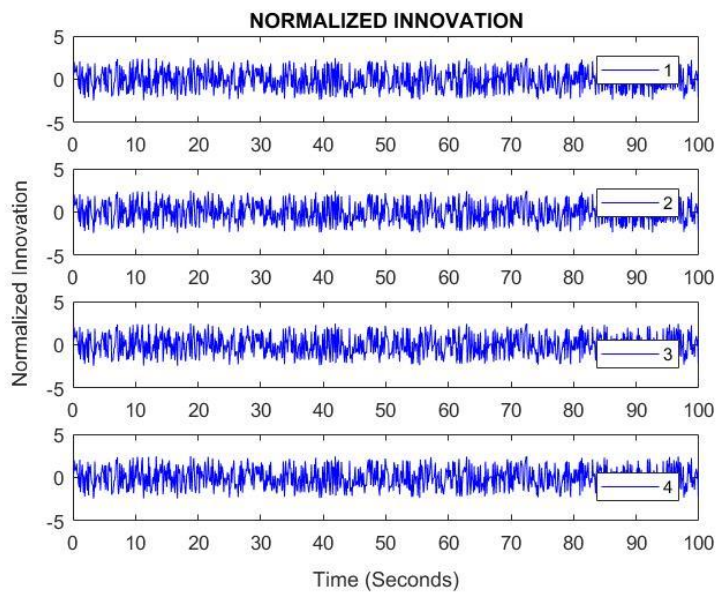

Fig.11: UKF Normalized Innovation Graphs

Root Mean Square Error (RMSE) tables are given below.

Table 1: RMSE for 20 Seconds Simulations

\begin{tabular}{|c|c|c|c|c|}
\hline & $\mathbf{X}(\mathbf{m})$ & $\mathbf{Y}(\mathbf{m})$ & $\mathbf{Z}(\mathbf{m})$ & Clock Bias (m) \\
\hline EKF & 4.0673 & 3.9843 & 5.3353 & 1.3685 \\
\hline UKF & 4.8352 & 3.7534 & 3.7852 & 1.0870 \\
\hline
\end{tabular}

Table 2: RMSE for 100 Seconds Simulations

\begin{tabular}{|c|c|c|c|c|}
\hline & $\mathbf{X}$ (m) & $\mathbf{Y}(\mathbf{m})$ & $\mathbf{Z}(\mathbf{m})$ & Clock Bias (m) \\
\hline EKF & 2.0290 & 1.7819 & 2.6600 & 1.3024 \\
\hline UKF & 1.8257 & 1.8651 & 1.9873 & 0.9056 \\
\hline
\end{tabular}


Table 3: RMSE for 200 Seconds Simulations

\begin{tabular}{|c|c|c|c|c|}
\hline & $\mathbf{X}(\mathbf{m})$ & $\mathbf{Y ~ ( m )}$ & $\mathbf{Z}(\mathbf{m})$ & Clock Bias (m) \\
\hline EKF & 1.5136 & 1.5253 & 1.9065 & 0.9605 \\
\hline UKF & 1.4070 & 1.3007 & 1.5967 & 0.8496 \\
\hline
\end{tabular}

Table 4: RMSE for 300 Seconds Simulations

\begin{tabular}{|c|c|c|c|c|}
\hline & $\mathbf{X}(\mathbf{m})$ & $\mathbf{Y}(\mathbf{m})$ & $\mathbf{Z}(\mathbf{m})$ & Clock Bias $(\mathbf{m})$ \\
\hline EKF & 1.2378 & 1.3821 & 1.6072 & 0.9069 \\
\hline UKF & 1.1968 & 1.1613 & 1.2945 & 0.6407 \\
\hline
\end{tabular}

Table 5: RMSE for 500 Seconds Simulations

\begin{tabular}{|c|c|c|c|c|}
\hline & $\mathbf{X}(\mathbf{m})$ & $\mathbf{Y}(\mathbf{m})$ & $\mathbf{Z}(\mathbf{m})$ & Clock Bias $(\mathbf{m})$ \\
\hline EKF & 1.1659 & 1.0109 & 1.2467 & 0.7086 \\
\hline UKF & 0.9169 & 0.9638 & 1.0230 & 0.6374 \\
\hline
\end{tabular}

By analysing the root mean square error tables, it's obtained that UKF estimations are better than EKF estimations for both short and long-time interval simulations. Also, when time of the simulation increased accuracy of the estimation increases. While root mean square errors are approximately 3-4 m for $20 \mathrm{sec}$ this value decreased below $1 \mathrm{~m}$ in $500 \mathrm{sec}$ simulations.

\section{Conclusion}

In this study, two different Kalman Filters, Extended and Unscented, have been simulated for stationary user. The position of the Şükrü SARAÇOĞLU Stadium (Istanbul/Turkey) estimated. Graphs and tables showed that UKF estimations achieved better results in both short-term and long-term analyzes compared to EKF. With this study, it has been revealed which method gives better results in determining the stationary user's position, and convenience is provided for future studies.

\section{References:}

[1] Bagci, M. \& Hajiyev, C. (2016). Integrated NRM/EKF for LEO satellite GPS based orbit determination. IEEE Metrology for Aerospace (MetroAeroSpace), Florence, 462-467.

[2] Erkec T.Y. and Hajiyev, C (2021), Satellite Formation Flight via NRM\&EKF State Estimation Method, International Symposium on Aircraft Technology, MRO \& Operations 2021, June 28-30June 2021, Budapest, Hungary.

[3] Erkec T.Y. and Hajiyev, C (2021), Satellite Formation Flight via NRM\&EKF State Estimation Method, International Symposium on Aircraft Technology, MRO \& Operations 2021, June 28-30June 2021, Budapest, Hungary.

[4] Erkec, T.Y., Hajiyev, C, (2021), Vision-Based Approaches of the Small Satellites Relative Navigation, WSEAS TRANSACTIONS on COMPUTER RESEARCH, DOI: 10.37394/232018.2021.9.3.

[5] Haciyev, Ç. (2010, Nisan). Deney Verilerinin İşlenme Yöntemleri ve Mühendislik Uygulamaları. Nobel Yayıncilık.

[6] Hajiyev, C. (2011). GNSS signal processing via linear and extended Kalman filters. Asian Journal of Control (Vol. 13, No.2, pp. 1-10).

[7] Julier, S., J., and Uhlmann, J., K. (1997, July). New extension of the Kalman filter to nonlinear systems. Proc. SPIE 3068. Signal Processing, Sensor Fusion, and Target Recognition VI. https://doi.org/10.1117/12.280797

[8] Kalman, R.E. (1960). A New Approach to Linear Filtering and Prediction Problems. Journal of Basic Engineering.

[9] Kumar, A. N., Suresh, C., Sasibhushana, R. G., (2018) Extended Kalman Filter for GPS Receiver Position Estimation. In: Bhateja V., Coello C., Satapathy S., Pattnaik P. (eds) Intelligent Engineering Informatics. Advances in Intelligent Systems and Computing, vol 695. Springer, Singapore.

[10] Kumar, A., \& Rao, G.S. (2019, May). Unscented Kalman Filter for GPS based positioning and tracking services. International Journal of Innovative Technology and Exploring Engineering (IJITEE). (Vol. 8)

[11] Sever M. and Hajiyev, C (2021), Comparison of GPS based position estimation methods, International Symposium on Aircraft Technology, MRO \& Operations 2021, June 2830June 2021, Budapest, Hungary.

[12] Sever M. and Hajiyev, C (2021), GPS Based Satellite Localization with Unscented Kalman Filter, International Symposium on Aircraft Technology, MRO \& Operations 2021, June 2830June 2021, Budapest, Hungary.

[13] Sever, M. \& Hajiyev, C. (2020, September). Satellite Localization Correction with Extended Kalman Filter. International Symposium on Electric Aviation and Autonomous Systems. 22$24 \mathrm{Kiev}$, Ukraine.

[14] Soken, H.E. \& Hajiyev, C. (2010). Pico satellite attitude estimation via robust unscented Kalman filter in the presence of measurement faults. ISA Trans., 49 (pp 249-256).

Creative Commons Attribution License 4.0 (Attribution 4.0 International, CC BY 4.0)

This article is published under the terms of the Creative Commons Attribution License 4.0

https://creativecommons.org/licenses/by/4.0/deed.en US 